\title{
Human Papillomavirus Detection and Abnormal Anal Cytology in HIV-infected Patients Using p16/Ki-67 Dual-Staining
}

\author{
Natcha Patarapadungkit ${ }^{1,2 *}$, Parinyabhorn Khonhan', Pornrith Pisuttimarn ${ }^{3}$, \\ Chamsai Pientong $^{2,4}$, Tipaya Ekalaksananan ${ }^{2,4}$, Supinda Koonmee ${ }^{1}$
}

\begin{abstract}
Objective: We investigated human papillomavirus (HPV) infection and detected anal squamous intraepithelial lesions by modified liquid-based cytology (LBC) and p16/Ki67 dual-staining. Methods: Anal swabs (n=393) were collected from patients with HIV infection. Anal cells were kept in 95\% ethyl alcohol for modified LBC. DNA was extracted from cells for HPV detection and genotyping using real-time PCR and reverse line blot hybridization. Results: Nine samples (2.3\%) were unsatisfactory specimens, 74.8\% (294/393) were negative for intraepithelial malignancies (NILM) and 22.9\% (90/393) exhibited squamous intraepithelial lesions (SIL). In the latter category, 13.7\% of samples (54/393) contained atypical squamous cells of undetermined significance (ASCUS), 6.9\% (27/393) were classified as low-grade SIL (LSIL) and 2.3\% (9/393) as high-grade SIL (HSIL). A total of 331 from 393 swab samples were suitable for detection of HPV infection. Among these, 34.1\% (113/331) were positive. HPV $58(15.9 \%)$ was the most common genotype, followed by HPV 18 (14.2\%) and HPV 16 (11.5\%). The severity of abnormal cells was significantly associated with HPV infection. Dual staining with p16/Ki-67 was performed on 130 samples: in 30.8\% (40/130) of samples positive staining was significantly associated with severity of abnormal cells. Agreement between cytology, p16/Ki67 dual-staining and high-risk HPV detection was 100\% in HSIL samples. Interestingly, eight apparently NIML cases might have contained abnormal cells, since they were positive by both p16/Ki67 dual-staining and high-risk HPV detection. Conclusion: Anal specimens screened using modified LBC with 95\% ethyl alcohol solution as the fixative are suitable for screening anal precancerous lesions by cytology, HPV testing and p16/Ki-67 dual staining.
\end{abstract}

Keywords: Anal cytology- HIV- HPV- immunocytochemistry- p16/ki67- dual-staining

Asian Pac J Cancer Prev, 21 (7), 2013-2019

\section{Introduction}

Anal cancer has striking similarities with cervical cancer in terms of etiology and pathogenesis. Both are associated with persistent infection by human papillomavirus (HPV) (Albuquerque and Medeiros, 2019). Immunosuppressed state with decreased numbers of CD4+ cells, HIV infection and sexual practices, such as receptive anal intercourse and high lifetime number of sexual partners, are all risk factors for anal HPV infection leading to anal cancer (Kelly et al., 2020). Over the past decade, the incidence rate of anal cancer has increased, particularly in $\mathrm{HIV}$-infected individuals among whom incidence is about 30 -fold higher than in the general population (Weis et al., 2011). The weakening immune system of HIV-infected individuals is an additional risk factor for HPV infection that leads to anal intraepithelial neoplasia (AIN) and anal cancer (van der Zee et al., 2013). Screening for cervical cancer by cytology is widely accepted as the best strategy to reduce incidence and mortality rate: anal cytology can perhaps do the same for anal cancer (Darragh and Winkler, 2011). Anal cytology screening is recommended for all at-risk populations, including HIV-infected individuals (Anthony et al., 2015). Anal cytology for detection of AIN is moderately sensitive (60-70\%) and specific (32-59\%). Additional tests need to be developed cytological technique for increase sensitive and specific to improve these results (Walts et al., 2014). Testing for HPV DNA is an adjunct to cytology, or can be used alone to screen for anal cancer: it has greater sensitivity (75-100\%) than cytology (Darragh and Winkler, 2011). However, HPV is very common in the general population and need not be associated with anal cancer. Hence this test is not very specific. In recent years, several biomarkers have been proposed for immunocytochemistry to detect expression of cellular products affected by HPV infection. Observed deregulation of the cell cycle identifies those infections at risk of progression toward dysplasia and carcinoma. Such

${ }^{1}$ Department of Pathology, Faculty of Medicine, Khon Kaen University, Khon Kaen, Thailand. ${ }^{2} H P V$ \& EBV and carcinogenesis Research Group, Khon Kaen University, Khon Kaen, Thailand. ${ }^{3}$ Sirindhorn Hospital, Khon Kaen, Thailand. ${ }^{4}$ Department of Microbiology, Faculty of Medicine, Khon Kaen University, Khon Kaen, Thailand. *For Correspondence: nuapat@kku.ac.th 
tests should be more specific than HPV DNA testing. One of the most widely studied markers is the cyclin-dependent kinase inhibitor p16 INK4a (p16) (Tay et al., 2017). Recently, a kit has been developed by CINtec ${ }^{\circledR}$ PLUS Cytology to stain for both p16 and Ki-67. Co-expression of p16 and $\mathrm{Ki}-67$ in the same anal cells is an indicator of a deregulated cell cycle caused by HPV oncoproteins and is typical of precancerous cells (Donà et al., 2012). There is limited information on the prevalence of anal HPV on the best strategy of screening for anal cancer. In this study, we investigated the correlation between results from cytology, HPV DNA and p16/Ki-67 dual staining in HIV-infected patients.

\section{Materials and Methods}

\section{Specimen collection and preparation}

The study protocol was reviewed and approved by the Ethics Committee of Khon Kaen University, Thailand (HE 551148). This study was a cross-sectional analysis of 393 HIV-infected individuals at Srinagarind Hospital and Sirindhorn Hospital in Thailand. Included were all such patients who underwent anal cancer screening between January 2014 and February 2016. Exclusion criteria were; presence of gastrointestinal hemorrhage, intestinal obstruction or anal wound. Anal specimens were collected with Rayon swabs that were gently inserted approximately 5-6 cm into the anal canal while rotating the swab to contact all sides of the anal canal. The anal swab was quickly placed in $95 \%$ ethyl alcohol prior to modified-liquid based cytology (Patarapadungkit et al., 2012).

\section{Anal cytology}

Anal cytology specimens in 95\% ethyl alcohol were centrifuged at $1500 \mathrm{rpm}$ for 10 minutes at room temperature. The sediment (approximately $200 \mu \mathrm{l}$ ) was transferred to positively charged slides. The slides were air-dried at room temperature then fixed in 95\% alcohol for at least $15 \mathrm{~min}$, and Papanicolaou stains were used to assess cellularity and morphology. All slides were evaluated independently by 2 cytologists. The cytology results were categorized following the Bethesda system (2014) as unsatisfactory specimen contains nucleated squamous cells less than 1-2 per high power field, many anucleated squamous epithelial cells, obscuring bacterial, fecal material and cellular debris, negative for intraepithelial lesion or malignancy (NILM), atypical squamous cells of undetermined significance (ASCUS), low-grade squamous intraepithelial lesion (LSIL), atypical squamous cells cannot rule out high-grade squamous intraepithelial lesion (ASC-H), High-grade squamous intraepithelial lesion (HSIL), and squamous cells carcinoma (SCC) (Nayar and Wilbur, 2015).

\section{HPV DNA extraction and genotyping}

Anal cells were washed with phosphate buffered saline by centrifugation at $13,500 \mathrm{rpm}, 4^{\circ} \mathrm{C}$ for 5 minutes and DNA was extracted using the AllPrep DNA mini kit (Qiagen, Valencia, USA) according to the manufacturer instruction. Quality of the extracted DNA was evaluated by amplification of the $\beta$-globin gene as an internal control. Samples positive by PCR for the $\beta$-globin gene were subjected to HPV DNA detection by real-time PCR on a LightCycler 480 instrument (Roche Diagnostics). A 142-bp fragment from the L1 region of HPV DNA was amplified using the consensus primers GP5+/GP6+ (van den Brule et al., 2002). The HPV DNA positive samples were used for HPV DNA genotyping by reverse line blot hybridization (RLBH), assaying $37 \mathrm{HPV}$ types including HPV 6, 11,16, 18, 26, 31, 33, 34, 35, 39, 40, 42, 43, 44, 45b, 51, 52, 53, 54, 55, 56, 57, 58, 59, 61, 66, 68, 70, 71 CP8106, 72, 73, 81 CP3804, 82 MM4, 82 IS39, 83 MM7, 84 MM8, CP 6108.

\section{Immunocytochemistry}

The residual specimens were centrifuged at 1,500 rpm for 10 minutes at room temperature, the sediment was aspirated approximately $200 \mu 1$ and then transferred to positively-charged slides. The slides were air-dried at room temperature after that fixed in $95 \%$ alcohol for at least 15 minutes and dried the slide on a flat horizontal surface for $60 \mathrm{~min}$ at room temperature, before performing p16/Ki-67 dual stain. The p16/Ki-67 dual stain study was performed automatically, using VENTANA BenchMark XT equipment and CINtec ${ }^{\circledR}$ PLUS Cytology Kit reagent. The kit designed were used a primary monoclonal mouse antibody directed to human p16 protein and a primary monoclonal rabbit antibody directed against Ki-67 protein. After counterstaining, a two-step mounting protocol must be applied: in the first step an aqueous mounting of the specimens using an aqueous mounting medium. Subsequently, the slides may be cover-slipped using a permanent mounting medium. The result can be evaluated by light microscopy inspection. The positive result of p16/Ki-67 was defined as one or more of anal epithelial cells stained with both a brown cytoplasmic stain (p16) and red nuclear (Ki-67) irrespective of interpretation of morphological abnormalities slides without any doublestained cells were called negative for p16/Ki-67 dual stain.

\section{Statistical analysis}

Statistical analysis was carried out using SPSS program, version 19.0 for Windows (SPSS, INc., Chicago, IL, USA). The chi-square test was used to quantify any correlation between anal cytology and HPV DNA testing. A value of $\mathrm{p}<0.05$ was considered statistically significant.

\section{Results}

\section{Cytological diagnosis}

In total, 393 anal specimens were collected including $64.6 \%(254 / 393)$ from males and $35.4 \%(139 / 393)$ from females. Nine of 393 specimens (2.3\%) were regarded as unsatisfactory in modified LBC: fewer than 6 nucleated squamous cells were present per high-power field. Among remaining samples, 74.8\% (294/393) were classified as negative for intraepithelial lesion or malignancy (NILM) and 22.9\% (90/393) as atypical squamous cells of undetermined significance (ASCUS) following the Bethesda system 2014. The most common categories within abnormal squamous intraepithelial lesion (ASIL) 


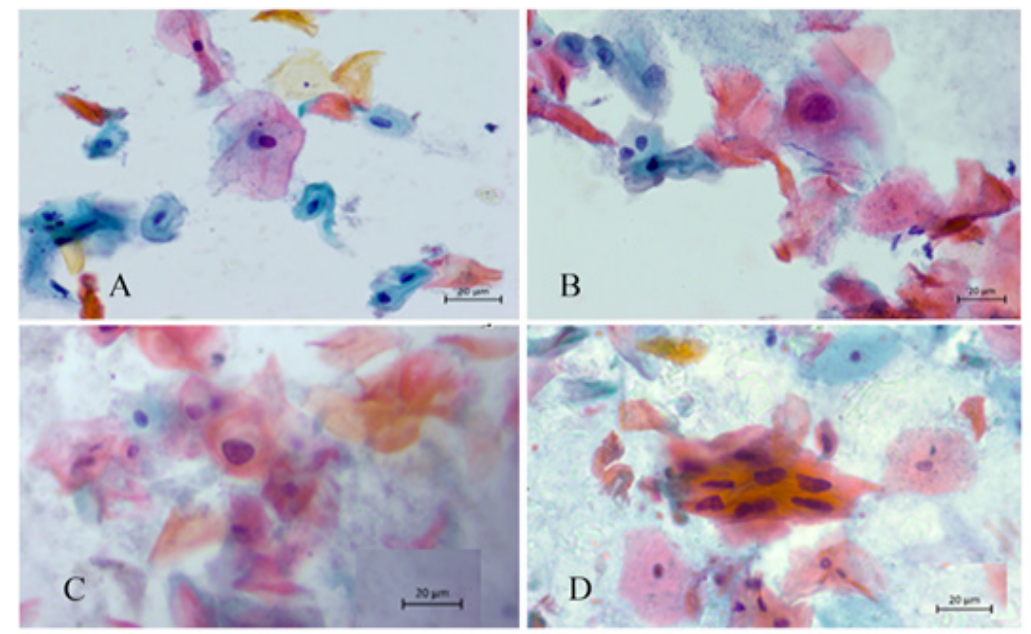

Figure 1. Representative Examples of modified LBC Pap Smear Showing: a. NILM, b. ASCUS, c. LSIL with HPV Infection with kiolocytes; and d. HSIL (arrow) from Papanicolaou stain (x400).

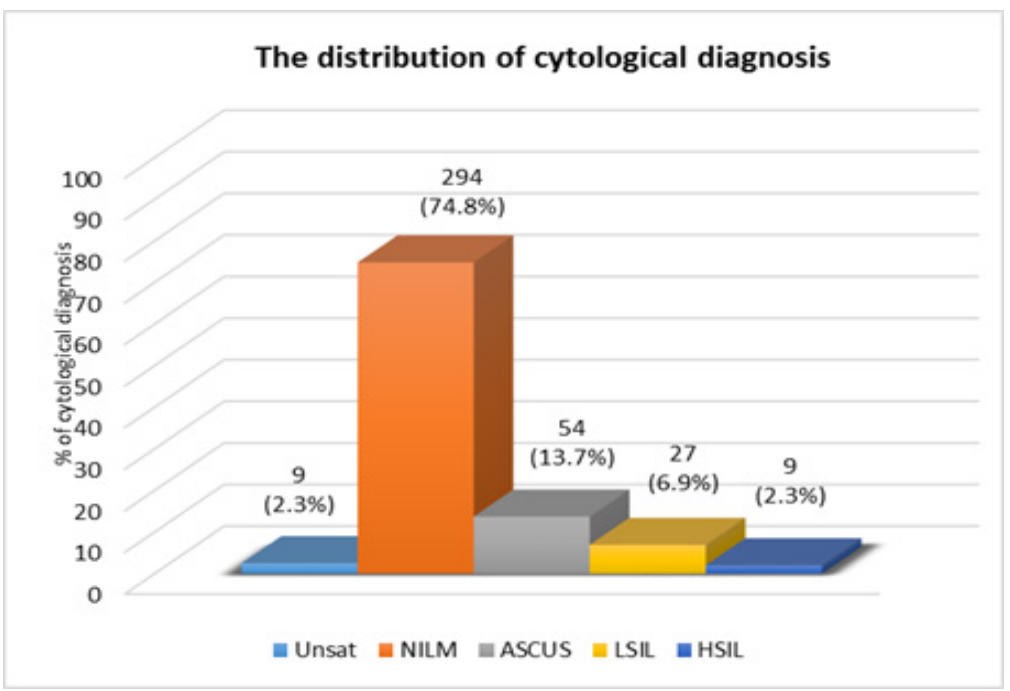

Figure 2. The Distribution of Cytological Diagnosis from HIV-Infected Patients 393 Cases

were ASCUS $(13.7 \%$; 54/393) followed by low-grade squamous intraepithelial lesion (LSIL) $(6.9 \% ; 27 / 393)$ and high-grade squamous intraepithelial lesion (HSIL) $(2.3 \%$; 9/393) (Figures 1, 2).

\section{The prevalence of HPV DNA}

Of the 393 anal specimens, valid HPV DNA results were obtained from 331: 62 samples lacking adequate material for DNA extraction were excluded. HPV DNA was not detected in $65.9 \%$ (248/331) of samples: $34.1 \%$ (113/331) were positive. The most common high-risk HPV genotypes in single-genotype infections were HPV $58(15.9 \% ; 18 / 113)$, HPV $18(14.2 \% ; 16 / 113)$ and HPV 16 $(11.5 \% ; 13 / 113)$. Other HPV high-risk genotypes present as single infections were HPV $33(1.8 \% ; 2 / 113)$, HPV 45 (0.9\%; 1/113), HPV $53(0.9 \% ; 1 / 113)$, HPV $56(0.9 \%$; 1/113), HPV $70(0.9 \% ; 1 / 113)$ and HPV $73(0.9 \% ; 1 / 113)$. Low-risk HPV genotypes detected were HPV 11 (6.2\%;

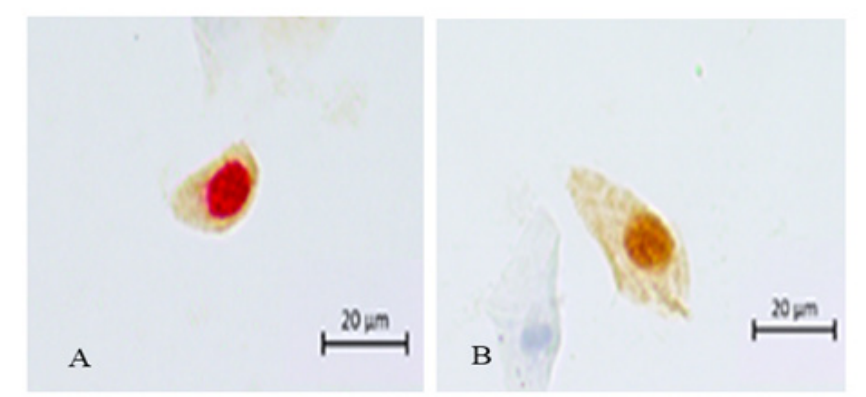

Figure 3 The p16/Ki-67 Dual Stain. HSIL cytology showed. a, Positive with red (Ki-67) staining nuclei and brown (p16) cytoplasm; b, Negative with brown (p16) both of nuclear and cytoplasm but no red (Ki-67) nuclear staining or no staining both of nuclear and cytoplasm 
Table 1. The Prevalence of HPV Detection and Genotyping in Cytological Diagnosis 113 Cases

\begin{tabular}{|c|c|c|c|c|c|}
\hline \multirow[t]{2}{*}{ HPV genotypes } & \multicolumn{4}{|c|}{ Cytological diagnosis } & \multirow{2}{*}{$\begin{array}{l}\text { Total } \\
\text { n (\%) }\end{array}$} \\
\hline & $\begin{array}{l}\text { NILM } \\
\mathrm{n}(\%)\end{array}$ & $\begin{array}{c}\text { ASCUS } \\
\mathrm{n}(\%)\end{array}$ & $\begin{array}{l}\text { LSIL } \\
\mathrm{n}(\%)\end{array}$ & $\begin{array}{l}\text { HSIL } \\
\mathrm{n}(\%)\end{array}$ & \\
\hline Single infection (any type) & $41 / 63(65.1)$ & $16 / 25(64.0)$ & $11 / 18(61.1)$ & $4 / 7(57.1)$ & $72 / 113(63.7)$ \\
\hline Any high-risk type & $37 / 63(58.7)$ & $9 / 25(36.0)$ & 6/18 (33.3) & $2 / 7(28.6)$ & $54 / 113(47.8)$ \\
\hline HPV 16 & $8(12.7)$ & $2(8.0)$ & $2(11.1)$ & $1(14.3)$ & $13(11.5)$ \\
\hline HPV 18 & $9(14.3)$ & $5(20.0)$ & $2(11.1)$ & - & $16(14.2)$ \\
\hline HPV 33 & $1(1.6)$ & - & $1(5.6)$ & - & $2(1.8)$ \\
\hline HPV 45 & $1(1.6)$ & - & - & - & $1(0.9)$ \\
\hline HPV 53 & $1(1.59)$ & - & - & - & $1(0.9)$ \\
\hline HPV 56 & - & - & - & $1(14.3)$ & $1(0.9)$ \\
\hline HPV 58 & $16(25.4)$ & $1(4.0)$ & $1(5.6)$ & - & $18(15.9)$ \\
\hline HPV 70 & $1(1.6)$ & - & - & - & $1(0.9)$ \\
\hline HPV 73 & - & $1(4.00)$ & - & - & $1(0.9)$ \\
\hline Any low-risk type & $4 / 63(6.4)$ & $7 / 25(28.0)$ & $5 / 18(27.8)$ & $2 / 7(28.6)$ & $18 / 113(15.9)$ \\
\hline HPV 6 & $1(1.6)$ & $3(12.0)$ & $1(5.6)$ & - & $5(4.4)$ \\
\hline HPV 11 & $2(3.2)$ & $2(8.0)$ & $2(11.1)$ & $1(14.3)$ & $7(6.2)$ \\
\hline HPV 42 & $1(1.6)$ & $1(4.0)$ & - & $1(14.3)$ & $3(2.7)$ \\
\hline HPV 43 & - & - & $1(5.6)$ & - & $1(0.9)$ \\
\hline HPV 72 & - & $1(4.0)$ & $1(5.6)$ & - & $2(1.8)$ \\
\hline Multiple infection & $11 / 63(17.5)$ & 6/25 (24.0) & $5 / 18(27.8)$ & $3 / 7(42.9)$ & $25 / 113(22.2)$ \\
\hline HPV 6,16 & - & $1(4.0)$ & - & $2(28.6)$ & $3(2.7)$ \\
\hline HPV 6,54 & $1(1.6)$ & - & - & - & $1(0.9)$ \\
\hline HPV 11,16 & $2(3.2)$ & - & - & - & $2(1.8)$ \\
\hline HPV 11,45 & - & - & $1(5.6)$ & - & $1(0.9)$ \\
\hline HPV 11,52 & $3(4.8)$ & $1(4.0)$ & $2(11.1)$ & - & $6(5.3)$ \\
\hline HPV 11,70 & - & - & $1(5.6)$ & - & $1(0.9)$ \\
\hline HPV 16,56 & - & - & $1(5.6)$ & - & $1(0.9)$ \\
\hline HPV 16,70 & - & $1(4.0)$ & - & - & $1(0.9)$ \\
\hline HPV 16,72 & - & $1(4.0)$ & - & - & $1(0.9)$ \\
\hline HPV 18,53 & $1(1.6)$ & - & - & - & $1(0.9)$ \\
\hline HPV 18,56 & - & $1(4.0)$ & - & - & $1(0.9)$ \\
\hline HPV 18,58 & $2(3.17)$ & - & - & - & $2(1.8))$ \\
\hline HPV 33,43 & - & - & - & $1(14.3)$ & $1(0.9)$ \\
\hline HPV 44,56 & - & $1(4.0)$ & - & - & $1(0.9)$ \\
\hline HPV 52,58 & $1(1.6)$ & - & - & - & $1(0.9)$ \\
\hline HPV 56,58 & $1(1.6)$ & - & - & - & $1(0.9)$ \\
\hline Undetermined & $11 / 63(17.5)$ & $3 / 25(12.0)$ & 2/18 (11.1) & - & $16 / 113(14.2)$ \\
\hline Total & $63(55.8)$ & $25(22.1)$ & $18(15.9)$ & $7(6.2)$ & 113 \\
\hline
\end{tabular}

7/113), HPV 6 (4.4\%; 5/113), HPV $42(2.7 \% ; 3 / 113)$, HPV $72(1.8 \% ; 2 / 113)$ and HPV $43(0.9 \% ; 1 / 113)$. The top three multiple-genotype HPV infections were HPV $11 \& 52(5.3 \% ; 6 / 113)$, HPV 6 \& $16(2.7 \% ; 3 / 113), \mathrm{HPV}$ $11 \& 16(1.8 \% ; 2 / 113)$ and HPV $18 \& 58(1.8 \% ; 2 / 113)$. HPV DNA was found in $55.8 \%(63 / 113)$ of specimens classed as NILM and in 44.2\% (50/113) of those classed as ASIL. In the latter category, HPV DNA was found in $22.1 \%(25 / 113)$ of ASCUS samples, $15.9 \%(18 / 113)$ of LSIL samples and 6.2\% (7/113) of HSIL samples (Table 1). The proportions of high-risk HPV infections did not differ significantly between samples classed as
ASCUS and those classed as LSIL $(\mathrm{p}=0.557)$. However, a significant difference in prevalence of high-risk HPV was found between samples classed as HSIL which will progress to cervical cancer $(\mathrm{p}=0.030)$.

p16/Ki-67 dual stain testing in cytological categories

Anal epithelial cells positive for Ki-67 showed red-stained nuclei while those positive for $\mathrm{p} 16$ had brown staining in the cytoplasm. The negative results of p16/Ki-67 dual stain were not stained or both of brown staining in the nuclear and cytoplasm of p16 (Figure 3). A total of 130 anal samples were detected by $\mathrm{p} 16 / \mathrm{Ki}-67$ 
Table 2. The Positive of p16/Ki-67 in Anal Cytology Categories

\begin{tabular}{lcccccc}
\hline HPV status & P16/Ki67 & NILM & ASCUS & LSIL & HSIL & Total \\
\hline HPV Negative (52) & negative (\%) & $32(100)$ & $13(81.3)$ & $1(25.0)$ & $0(0.0)$ & $46(88.5)$ \\
& positive (\%) & $0(0.0)$ & $3(18.7)$ & $3(75.0)$ & $0(0.0)$ & $6(11.5)$ \\
HR HPV positive (49) & negative (\%) & $19(70.4)$ & $7(63.6)$ & $3(42.9)$ & $0(0.0)$ & $29(59.2)$ \\
& positive (\%) & $8(29.6)$ & $4(36.4)$ & $4(57.1)$ & $4(100.0)$ & $20(40.8)$ \\
LR HPV positive (17) & negative (\%) & $3(100.0)$ & $5(71.4)$ & $0(0.0)$ & $1(50.0)$ & $9(52.9)$ \\
& positive (\%) & $0(0.0)$ & $2(28.6)$ & $5(100.0)$ & $1(50.0)$ & $8(47.1)$ \\
Undetermined (12) & negative (\%) & $5(71.4)$ & $1(50.0)$ & $0(0.0)$ & $0(0.0)$ & $6(50.0)$ \\
& positive (\%) & $2(28.6)$ & $1(50.0)$ & $2(100.0)$ & $1(100.0)$ & $6(50.0)$ \\
Total & & $(53.1) 69$ & $(27.7) 36$ & $(13.8) 18$ & $(5.4) 7$ & 130 \\
\hline
\end{tabular}

dual stain testing. The 52 negative HPV results were found $100 \%(32 / 32)$ in NILM, but were found positive p16/Ki-67in $18.75 \%(3 / 16)$ of ASCUS and $75 \%(3 / 4)$ of LSIL. All HSIL samples positive for high-risk HPV and Undetermined were also positive for p16/Ki-67. Corresponding values for LSIL, ASCUS and NILM samples were $57.1 \%, 36.4 \%$ and $29.6 \%$, respectively. Low-risk HPV samples were all negative p16/Ki-67 in NILM, but were stained positive for $28.6 \%$ (2/7) ASIL; $100 \%(5 / 5)$ LSIL and 50\% (1/2) HSIL. The undetermined HPV were positive p16/Ki-67 in both NILM and ASIL which were stained in $100 \%$ of LSIL and HSIL (Table 2).

\section{Discussion}

Screening for anal cancer is recommended for all HIV-infected patients, although no standard guidelines exist. Following the cervical cancer screening model, anal cytology has been proposed for anal cancer screening (Ehrenpreis and Smith, 2018). In this regard, the conventional smear is often air-dry artifact. Liquid-based cytology preparations such as ThinPrep, Liqui-PREP and modified liquid-base cytology yield low background smears due to reduction in fecal material and bacteria, facilitating easier visualization of abnormal cells (Patarapadungkit et al., 2012; Pankam et al., 2017; Khattab et al., 2018). We found abnormal anal cytology in $22.9 \%$ of HIV-infected patients. Another study in Thailand reported the prevalence of ASIL among HIV-infected men who have sex with men (MSM) to be $43.0 \%$ (Ruanpeng et al., 2016). Among samples in the ASIL category, 13.7\% were in the ASCUS subcategory, a lower proportion than reported in another study on HIV-infected MSM (between 16.7\% and 25.7\%) (Weis et al., 2011). We classified $2.3 \%$ of samples as HSIL, which is considered the real precursor of anal carcinoma. LSIL, at $6.9 \%$, was a more frequent diagnosis than HSIL. It is worth noting that LSIL might be clinically important. In one study, about $62 \%$ of HIV-positive MSM and $36 \%$ of HIVnegative MSM who had a diagnosis of LSIL at baseline progressed to HSIL within 2-4 years (Palefsky et al., 1998). A diagnosis of ASCUS may also hide squamous intraepithelial lesions (Wang et al., 2019). The presence of any abnormal anal cytological finding indicates a potential association with high-grade anal intraepithelial neoplasia (AIN) on pathological examination (Machalek et al., 2016). These data underline that all patients with a cytological report of ASCUS or worse need a follow-up for accurate diagnostic investigation. Importantly, HPV is the most common sexually transmitted viral disease implicated in progression of anal intraepithelial lesion into squamous cell carcinoma. HPV has a particularly high prevalence among HIV-infected patients. HPV DNA testing is recommended an alternative or an adjunct to cytology (Dias et al., 2019). In our anal samples collected for modified LBC, the prevalence of HPV infection was $34.14 \%$, which lies within the range noted in previous publications (Gandra et al., 2015; Stier et al., 2015). It should be noted that the prevalence of ASIL and HPV infection varies according to sexual behavior and geography. Furthermore, infection by any HPV is associated with a 4-fold increased risk of having abnormal anal cytology (Donà, et al., 2012). In our study, the prevalence of HPV infection seems to rise with grade of cytological abnormality. We found HPV infection, both with single and multiple genotypes, in all ASIL samples. Infections with two or more HPV genotypes is common in anal specimens (Dietrich et al., 2015). The prevalence of high-risk HPV single infection was $47.8 \%$. Corresponding values for women and for HIV-infected men who have sex with men range from 37.5\% (Fan et al., 2020). Among high-risk HPV types, we found HPV 58 to be the most common, followed by HPV 18, HPV 16 and HPV 33. The most common high-risk HPV types in HIV patients worldwide are HPV 16 followed by HPV 18 (Fan et al., 2020; Nasioutziki et al., 2020). Among Spanish MSM, the most prevalent high-risk HPV was HPV 31 and in China, HPV 58 were the most frequently detected (Torres et al., 2013; Liu et al., 2019). As for low-risk HPV types, HPV 11, HPV 6 and HPV 42 were the most frequently detected. Abnormal anal cytology can be associated with low-risk HPV types HPV6, HPV 11, HPV 40 and HPV 42 (Zhang et al., 2014). The frequency of HPV genotypes varies by geographic region. The high-risk HPV infections were detected in cases with each cytological diagnosis including negative, ASCUS, LSIL and HSIL. However, it should be noted that HPV DNA testing has high sensitivity but low specificity as a screening tool for cancer (Kang et al., 2020). The presence of high-risk HPV infection in cases classed as ASCUS may reflect the fact that a diagnosis of ASCUS is consistent with benign reactive changes other than malignancy. Similarly, in cases classed as LSIL by 
cytology, we found infection with high-risk HPV. LSIL may be a consequence of low-risk HPV infection and other causes. HSIL cases were positive for HPV DNA including high-risk HPV, low-risk HPV and multiple infections with both. We found HSIL to be strongly associated with highrisk HPV infection, as also reported by others (Dietrich et al., 2015). In the immunocytochemistry study, we conducted p16/Ki-67 dual staining on a selected series of anal specimens with all grades of abnormal cytology and/ or HPV DNA-positive. We investigated the correlation between p16/Ki-67 dual staining and the cytological diagnosis and high-risk HPV infection. There was a significant increase in samples positive for $\mathrm{p} 16 / \mathrm{Ki}-67$ with increasing severity of the cytological lesion, especially in HISL with high-risk HPV infection. Nevertheless, we found 8 cases $(29.63 \%)$ of cytologically normal samples with high-risk HPV infection and positive for p16/Ki-67 staining. It may be that these 8 cases are also precancerous despite their normal appearance. Similarly, Lee and Lee (2016) demonstrated that 5 of 6 cervical samples of ASCUS and LSIL with p16 methylation progressed to HSIL (Lee and Lee, 2016). It is worth noting that 8 of 17 cases $(47.06 \%)$ of ASIL that were positive for low-risk HPV were also positive for $\mathrm{p} 16 / \mathrm{Ki}$ 67. One follow-up study on cervical cases classified as ASCUS and negative for high-risk HPV found that, one year later, $23 \%$ had progressed to LSIL and $4 \%$ to HSIL (Ozturk et al., 2016). Furthermore, Balmagambetova et al., (2019), in a review of the literature, noted the reported presence of low-risk HPV types 6 and 11 in invasive anal cancer cases (Balmagambetova et al., 2019) and Serrano et al., (2015) reported low-risk HPV associated with cancers of the anus, cervix, vagina and vulva (Serrano et al., 2015). Several previous studies have reported p16/Ki-67 positivity is independent of HPV genotypes and achieved a sensitivity equal to HPV testing with significantly higher specificity (Zhang et al., 2019; Hu et al., 2020). Consequently, the presence of p16/Ki-67 staining in normal cells or dysplastic cells should not be ignored and may indicate deregulation of the cell cycle. In this study, we detected p16/Ki-67 dual stain in anal specimens positive for HPV or cytological diagnosis as ASIL including ASCUS, LSIL and HSIL. Such staining was not detected in NILM samples positive for low-risk HPV or without HPV. Therefore, the screening of anal samples with p16/Ki-67 dual stain should be done when the patient has a cytological diagnosis of ASCUS or LSIL, with or without HPV infection. Limitations of our study were that a small number of cases were analyzed by $\mathrm{p} 16 /$ Ki-67 dual staining and no biopsy was taken to confirm specificity.

In conclusion, anal specimens screened using modified LBC with 95\% ethyl alcohol solution as the fixative are suitable for screening anal precancerous lesions by cytology, HPV testing and p16/Ki-67 dual staining. This study should contribute to management guidelines for anal cancer screening. If the results of cytology were ASCUS and LSIL, with or without the presence of HPV DNA, further investigation by p16/Ki-67 dual staining is worthwhile.

\section{Acknowledgements}

The authors thank the patients and their families for their participation and staff in the cytology lab for their assistance. This study was funded by Khon Kaen University, Thailand (Grant Number 581202). Roche Diagnostics (Thailand) Ltd. donated the CINtec $®$ PLUS Kit (Ventana Medical Systems, Inc). We would like to acknowledge Prof. David Blair, for editing the MS via the Publication Clinic KKU, Thailand.

Ethical approval The research was given ethical approval by the Khon Kaen University Ethics Committee (No. HE 551148).

\section{References}

Albuquerque A, Medeiros R (2019). New insights into the role of human papillomavirus in anal cancer and anal wart development. Acta Cytol, 63, 118-23.

Anthony P, Feinn R, Brenner B, et al (2015). The addition of high-risk HPV testing to anal cytology increases the identification of anal dysplasia in HIV-infected patients. Conn Med, 79, 389-94.

Balmagambetova SK, Tinelli A, Urazayev ON, et al (2019). HPV types distribution in general female population and in women diagnosed with cervical cancer across western Kazakhstan. Asian Pac J Cancer Prev, 20, 1089-96.

Darragh TM, Winkler B (2011). Anal cancer and cervical cancer screening: Key differences. Cancer Cytopathol, 119, 5-19.

Dias Gonçalves Lima F, Viset JD, Leeflang MMG, et al (2019) The accuracy of anal swab-based tests to detect high-grade anal intraepithelial neoplasia in HIV-infected patients: a systematic review and meta-analysis. Open Forum Infect Dis, 6, ofz191.

Dietrich A, Hermans C, Heppt MV, et al (2015). Human papillomavirus status, anal cytology and histopathological outcome in HIV-positive patients. J Eur Acad Dermatol Venereol, 29, 2011-8.

Donà $\mathrm{MG}$, Benevolo $\mathrm{M}$, Vocaturo $\mathrm{A}$, et al (2012). Anal cytological abnormalities and epidemiological correlates among men who have sex with men at risk for HIV-1 infection. BMC Cancer, 12, 476.

Donà MG, Vocaturo A, Giuliani M, et al (2012). p16/Ki-67 dual staining in cervico-vaginal cytology: correlation with histology, Human Papillomavirus detection and genotyping in women undergoing colposcopy. Gynecol Oncol, 126, 198-202.

Ehrenpreis ED, Smith DG (2018). Patients with newly diagnosed cervical cancer should be screened for anal human papilloma virus and anal dysplasia: Results of a pilot study using a STELLA computer simulation and economic model. Papillomavirus Res, 5, 38-45.

Fan S, Li P, Ouyang L, et al (2020). Anal human papillomavirus infection among MSM Attending University in China: Implications for Vaccination. Vaccines (Basel), 8, pii: E175.

Gandra S, Azar A, Wessolossky M (2015). Anal high-risk human papillomavirus infection and high-grade anal intraepithelial neoplasia detected in women and heterosexual men infected with human immunodeficiency virus. HIV AIDS Auckl NZ, 7, 29-34.

Hu Y, Hong Z, Gu L, et al (2020). Evaluation of p16/Ki-67 dualstained cytology in triaging HPV-positive women during cervical cancer screening. Cancer Epidemiol Biomarkers Prev, doi: 10.1158/1055-9965.EPI-19-1180.

Lee H, Lee E-J (2016). HPV infection and p16 promoter methylation as predictors of ASC-US/LSIL progression. 
Cancer Cytopathol, 124, 58-65.

Liu X, Lin H, Chen X, et al (2019). Prevalence and genotypes of anal human papillomavirus infection among HIV-positive vs. HIV-negative men in Taizhou, China. Epidemiol Infect, 147, e117.

Kang M, Ha SY, Cho HY, et al (2020). Comparison of papanicolaou smear and human papillomavirus (HPV) test as cervical screening tools: can we rely on HPV test alone as a screening method? An 11-year retrospective experience at a single institution. J Pathol Transl Med, 54, 112-8.

Kelly H, Chikandiwa A, Alemany Vilches L, et al (2020). Association of antiretroviral therapy with anal high-risk human papillomavirus, anal intraepithelial neoplasia, and anal cancer in people living with HIV: a systematic review and meta-analysis. Lancet HIV, 7, e262-78.

Khattab R, McMeekin E, Taege AJ, et al (2018). Unsatisfactory exfoliative anal cytology samples, 15-year experience with histologic, cytologic, and molecular follow-up. Diagn Cytopathol, 46, 117-21.

Machalek DA, Jin F, Poynten IM, et al (2016) Prevalence and risk factors associated with high-grade anal squamous intraepithelial lesions (HSIL)-AIN2 and HSIL-AIN3 in homosexual men. Papillomavirus Res, 2, 97-105.

Nasioutziki M, Chatzistamatiou K, Loufopoulos PD, et al (2020). Cervical, anal and oral HPV detection and HPV type concordance among women referred for colposcopy. Infect Agent Cancer, 15, doi: 10.1186/s13027-020-00287-7.

Nayar R, Wilbur DC (2015). The Pap Test and Bethesda 2014: "The reports of my demise have been greatly exaggerated. (after a quotation from Mark Twain)". J Low Genit Tract Dis, 19, 175-84.

Ozturk M, Umudum H, Aydin A, et al (2016). Immediate risk of HSIL presence in women who have both ASC-US cytology and negative high-risk HPV test. Eur J Gynaecol Oncol, 37, 232-7.

Patarapadungkit N, Koonmee S, Pasatung E, et al (2012). Anal cancer screening by modified liquid-based cytology in an HIV clinic. Asian Pac J Cancer Prev, 13, 4487-90.

Pankam T, Kerr SJ, Teeratakulpisan N, et al (2017). Human papillomavirus in anal biopsy tissues and liquid-based cytology samples of HIV-positive and HIV-negative Thai men who have sex with men. Papillomavirus Res, 3, 149-54.

Ruanpeng D, Chariyalertsak S, Kaewpoowat Q, et al (2016). Cytological anal squamous intraepithelial lesions associated with anal high-risk human papillomavirus infections among men who have sex with men in Northern Thailand. PLoS One, 11, e0156280.

Serrano B, de Sanjosé S, Tous S, et al (2015). Human papillomavirus genotype attribution for HPVs $6,11,16$, 18, 31, 33, 45, 52 and 58 in female anogenital lesions. Eur J Cancer, 51, 1732-41.

Stier EA, Sebring MC, Mendez AE, et al (2015). Prevalence of anal human papillomavirus infection and anal HPV-related disorders in women: a systematic review. Am J Obstet Gynecol, 213, 278-309.

Tay TKY, Lim KL, Hilmy MH, et al (2017). Comparison of the sensitivity and specificity of p16/Ki-67 dual staining and HPV DNA testing of abnormal cervical cytology in the detection of histology proven cervical intraepithelial neoplasia grade 2 and above (CIN 2+). Malays J Pathol, 39, 257-65.

Torres M, González C, del Romero J, et al (2013). Anal human papillomavirus genotype distribution in HIV-infected men who have sex with men by geographical origin, age, and cytological status in a Spanish cohort. J Clin Microbiol, 51, 3512-20.

van den Brule AJ, Pol R, Fransen-Daalmeijer N, et al (2002).
GP5+/6+ PCR followed by reverse line blot analysis enables rapid and high-throughput identification of human papillomavirus genotypes. J Clin Microbiol, 40, 779-87.

Van der Zee RP, Richel O, de Vries HJC, et al (2013). The increasing incidence of anal cancer: can it be explained by trends in risk groups. Neth J Med, 71, 401-11.

Walts AE, Manna P, Chan RC, et al (2014). Does high-risk human papilloma virus genotyping of abnormal anal cytology improve detection of high-grade anal intraepithelial neoplasia. J American Soc Cytopatho, 3, 236-43.

Wang YY, Kong LH, Liu Y, et al (2019). Retrospective analysis of cervical cancer and precancerous lesions in patients with atypical squamous cells of undetermined significance in China. Medicine (Baltimore), 98, e18239.

Weis SE, Vecino I, Pogoda JM, Susa JS, et al (2011). Prevalence of anal intraepithelial neoplasia defined by anal cytology screening and high-resolution anoscopy in a primary care population of HIV-infected men and women. Dis Colon Rectum, 54, 433-41.

Zhang DY, Yin YP, Feng TJ, et al (2014). HPV infections among MSM in Shenzhen, China. PLoS One, 9, e96364.

Zhang SK, Jia MM, Zhao DM, et al (2019). Evaluation of p16/ Ki-67 dual staining in the detection of cervical precancer and cancer in China. Cancer Epidemiol, 59, 123-8.

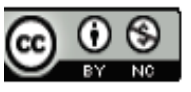

This work is licensed under a Creative Commons AttributionNon Commercial 4.0 International License. 